\title{
Rational points on certain elliptic surfaces
}

\author{
by \\ Maciej Ulas (Kraków)
}

Dedicated to Professor A. Schinzel on the occasion of his 70th birthday

1. Introduction. Let $\mathcal{E}$ be an elliptic surface given by the equation

$$
\mathcal{E}: y^{2} z=x^{3}+A(t) x z^{2}+B(t) z^{3},
$$

where $A, B \in \mathbb{Q}[t]$. The discriminant for $\mathcal{E}$ is defined by $\Delta(t)=-16\left(4 A(t)^{3}+\right.$ $\left.27 B(t)^{2}\right)$, while the $j$-invariant is $j(t)=-1728(4 A(t))^{3} / \Delta(t)$. We call the surface $\mathcal{E}$ isotrivial if its $j$-invariant is constant. We say that $\mathcal{E}$ splits if there exists an elliptic curve $E$ such that $\mathcal{E} \simeq E \times \mathbb{P}$ over $\mathbb{C}$. In what follows, by an elliptic surface we mean a non-split one. There is a natural projection on $\mathcal{E}$ given by $\pi: \mathcal{E} \ni([x: y: z], t) \mapsto t \in \mathbb{P}$. The mapping $\sigma: \mathbb{P} \rightarrow \mathcal{E}$ satisfying $\pi \circ \sigma=\mathrm{id}_{\mathbb{P}}$ will be called a section on $\mathcal{E}$. Throughout the paper, by a section we mean one defined over $\mathbb{Q}$. Note that we always have the zero section on $\mathcal{E}$ given by $\sigma_{0}=([0: 1: 0], t)$. We can look at the surface $\mathcal{E}$ as an elliptic curve defined over $\mathbb{Q}(t)$. Hence, we have the Mordell-Weil type theorem for $\mathcal{E}$, which says that the set of sections (or equivalently points on $\mathcal{E}$ defined over $\mathbb{Q}(t)$ ) forms a finitely generated abelian group.

Since for all but finitely many $t \in \mathbb{Q}$ the fibre $\mathcal{E}_{t}$ of the mapping $\pi$ is an elliptic curve, a natural question arises: what can we say about the set of $t \in \mathbb{Q}$ such that the elliptic curve $\mathcal{E}_{t}$ has a positive rank? If $\mathcal{E}$ has a non-torsion section, an answer to this question follows trivially from Silverman's specialization theorem ([12, p. 368]). It says that for all but finitely many $t \in \mathbb{Q}$ the curve $\mathcal{E}_{t}$ has a positive rank. Another interesting question concerns the existence of rational curves on $\mathcal{E}$. Note that each such curve, say $(x(u), y(u), \psi(u))$, yields a rational base change $t=\psi(u)$ such that $\sigma=$ $(x(u), y(u))$ is a section on the surface $\mathcal{E}_{\psi}: y^{2}=x^{3}+A(\psi(u)) x+B(\psi(u))$. We will see that in many cases $\sigma$ is a non-torsion section. A problem of this

2000 Mathematics Subject Classification: Primary 11D25; Secondary 11 G05.

Key words and phrases: diophantine equations, elliptic surfaces.

The author is a scholarship holder of the Stanisław Estreicher Fund. 
kind was considered in Whitehead's paper [13]. He proved that there exists a rational curve on the surface given by the equation $z^{2}=f(x, y)$, where $f \in \mathbb{Q}[x, y]$ and $\operatorname{deg} f=3$. It is easy to see that such a surface is birationally equivalent to $\mathcal{E}$ for some $A, B \in \mathbb{Q}[t]$ with $\operatorname{deg} A \leq 2, \operatorname{deg} B \leq 3$.

Also note that the existence of a rational base change $t=\psi(u)$ such that $\mathcal{E}_{\psi}$ has a non-torsion section, and Silverman's specialization theorem, imply that for all but finitely many $u \in \mathbb{Q}$, each fibre $\mathcal{E}_{\psi(u)}$ has a dense set of rational points. This also proves that the set of rational points on $\mathcal{E}$ is dense in the Zariski topology.

In Section 2 we consider a surface of the form $\mathcal{E}_{f}: y^{2}=x^{3}+f(t) x$, where $f \in \mathbb{Q}[t]$ and $\operatorname{deg} f \leq 4$. If $\operatorname{deg} f \leq 3$, then we show that there exists a rational base change $t=\varphi(s)$ such that the surface $\mathcal{E}_{f \circ \varphi}$ has a non-torsion section. A similar theorem is proved for $\operatorname{deg} f=4$ under the assumption that there exists $t_{0} \in \mathbb{Q}$ such that there are infinitely many rational points on the curve $E_{t_{0}}: y^{2}=x^{3}+f\left(t_{0}\right) x$. In particular, we prove that if $f$ of degree four is not even, then there is a non-trivial rational point on the surface $\mathcal{E}_{f}$.

In Section 3 we consider a surface of the form $\mathcal{E}^{g}: y^{2}=x^{3}+g(t)$, where $g \in \mathbb{Q}[t]$ is a monic polynomial of degree six. We prove that if $g$ is not even, then there is a rational base change $t=\chi(u)$ such that $\mathcal{E}^{g \circ \chi}$ has a non-torsion section. Moreover, if $g$ is even, and there exists $t_{0} \in \mathbb{Q}$ such that the curve $E^{t_{0}}: y^{2}=x^{3}+g\left(t_{0}\right)$ contains infinitely many rational points, then the set of $t_{0} \in \mathbb{Q}$ such that $E^{t_{0}}$ has a positive rank is infinite.

In Section 4 we present some results concerning diophantine equations of the form

$$
x^{2}-y^{3}-g(z)=t,
$$

where $g(z)=z^{6}+a z^{4}+b z^{3}+c z^{2}+d z+e \in \mathbb{Z}[z]$ and $t$ is a variable. We will deal with solutions of this equation in the ring of polynomials $\mathbb{Q}[t]$. In particular, we prove that if $a \equiv 1(\bmod 2)$ and $b \neq 0$, then the above equation has infinitely many solutions in $\mathbb{Q}[t]$.

In Section 5 we give some results about rational points on certain nonisotrivial elliptic surfaces.

REMARK 1.1. Most of the elliptic surfaces considered in this paper are geometrically rational, in other words, rational over $\mathbb{C}$. The referee suggested approaching our issues with the use of the theory of Mordell-Weil lattices for rational elliptic surfaces [11], [9]. This theory shows that $r(\mathcal{E})=$ $\operatorname{rank}(\mathcal{E}(\mathbb{C}(t))) \leq 8$. Moreover, for generators $\left(x_{1}, y_{1}\right), \ldots,\left(x_{n}, y_{n}\right)$ of the Mordell-Weil group of $\mathcal{E}(\mathbb{C}(t))$ we know that $x_{i} \in K[t]$ and $\operatorname{deg} x_{i}(t) \leq 2$, where $K$ is a certain finite extension of $\mathbb{Q}$. So, we can try to find such generators if $r(\mathcal{E})>0$ and next consider the Galois invariant part of the group $\mathcal{E}(K(t))$. Unfortunately, in a general situation (for instance, the one considered in this work) it is practically unfeasible, because it requires the 
examination of extensions of $\mathbb{Q}$ by roots of polynomial equations of degree $>3$ with variable coefficients. To the best of the author's knowledge the only work where this method was used and all calculations are conducted is the work by A. Bremner [1]. He considers the elliptic surface $y^{2}=x^{3}+f(t)$, where $f \in \mathbb{Q}[t]$ and $\operatorname{deg} f \leq 3$. Already in this case, when we have only three variable coefficients, the solutions get more and more complex and it is difficult to devise how the method can be generalized.

2. Rational points on $\mathcal{E}_{f}: y^{2}=x^{3}+f(t) x$. Let $f \in \mathbb{Q}[t] \backslash \mathbb{Q}$ and assume that $\operatorname{deg} f \leq 4$ and $f$ has at least two different complex roots. We consider the surface $\mathcal{E}_{f}$ given by the equation

$$
\mathcal{E}_{f}: y^{2}=x^{3}+f(t) x .
$$

Since $f$ does not have a root of multiplicity four, $\mathcal{E}_{f}$ is non-split. For a given $t \in \mathbb{Q}$ denote the curve $y^{2}=x^{3}+f(t) x$ by $E_{t}$. Note that for fixed $t \in \mathbb{Q}$, the torsion part of the group $E_{t}(\mathbb{Q})$ is isomorphic to one of the following $([12$, p. 323$]): \mathbb{Z} / 4 \mathbb{Z}$ if $f(t)=4 ; \mathbb{Z} / 2 \mathbb{Z} \times \mathbb{Z} / 2 \mathbb{Z}$ if $-f(t)$ is a square; $\mathbb{Z} / 2 \mathbb{Z}$ otherwise. As an immediate consequence we find that if there is a rational base change $t=\beta(u)$ such that the surface $\mathcal{E}_{f \circ \beta}$ has a section $\sigma=(x, y)$ with $y \neq 0$, then $\sigma$ is non-torsion.

We show the following

THEOREM 2.1.

(1) If $\operatorname{deg} f \leq 3$, then there exists a rational base change $t=\varphi(s)$ such that there is a non-torsion section on the surface $\mathcal{E}_{f \circ \varphi}$.

(2) If $\operatorname{deg} f=4$ and there is $t_{0} \in \mathbb{Q}$ such that the curve $E_{t_{0}}$ has infinitely many rational points, then there exists a rational base change $t=$ $\psi(r)$ such that there is a non-torsion section on $\mathcal{E}_{f \circ \psi}$.

Proof. It will be convenient to work with the surface $\mathcal{E}_{f}^{\prime}$ given by the equation

$$
\mathcal{E}_{f}^{\prime}: X Y^{2}=X^{2}+f(t)
$$

which is birationally equivalent to $\mathcal{E}_{f}$ via the mapping $(x, y, t)=(X, X Y, t)$ with inverse $(X, Y, t)=(x, y / x, t)$. Set $F(X, Y, t):=X Y^{2}-X^{2}-f(t)$.

Proof of (1). Let $f \in \mathbb{Q}[t]$ and $\operatorname{deg} f \leq 3$. Without loss of generality we can assume that $f(t)=a t^{3}+b t^{2}+c t+d$ for some $a, b, c, d \in \mathbb{Z}$ with $a \neq 0$ or $b \neq 0$. If $a=b=0$, then $f$ has degree 1 and if we put $t=\left(s^{4}-d\right) / c$, the surface splits over $\mathbb{Q}(s)$. Set $X=p T+q, Y=r T+s, t=T$. For these $X, Y, t$ we obtain

$$
F(X, Y, t)=a_{0}+a_{1} T+a_{2} T^{2}+a_{3} T^{3},
$$

where 


$$
\begin{array}{ll}
a_{0}=-d-q^{2}+q s^{2}, & a_{1}=-c-2 p q+2 q r s+p s^{2}, \\
a_{2}=-b-p^{2}+q r^{2}+2 p r s, & a_{3}=-a+p r^{2} .
\end{array}
$$

Note that the system of equations $a_{2}=a_{3}=0$ has exactly one solution given by

$$
p=\frac{a}{r^{2}}, \quad q=\frac{a^{2}+b r^{4}-2 a r^{3} s}{r^{6}} .
$$

For these $p, q$ the equation $F(p T+q, r T+s, T)=0$ has a root $T=$ $-\varphi_{1}(r, s) / \varphi_{2}(r, s)$, where

$$
\begin{aligned}
\varphi_{1}(r, s)= & a^{4}+2 a^{2} b r^{4}+b^{2} r^{8}+d r^{12}-4 a r^{3}\left(a^{2} s+b r^{4}\right) s \\
& +r^{6}\left(3 a^{2}-b r^{4}\right) s^{2}+2 a r^{9} s^{3}, \\
\varphi_{2}(r, s)= & r^{4}\left(2 a^{3}+2 a b r^{4}+c r^{8}-2 r^{3}\left(3 a^{2}+b r^{4}\right) s+3 a r^{6} s^{2}\right) .
\end{aligned}
$$

We have obtained a two-parameter solution of the equation defining the surface $\mathcal{E}_{f}^{\prime}$. If we define $\varphi(s):=-\varphi_{1}(1, s) / \varphi_{2}(1, s)$ and $t=\varphi(s)$, then $\sigma=$ $(p \varphi(s)+q,(p \varphi(s)+q)(\varphi(s)+s))$ is a section on the surface $\mathcal{E}_{f \circ \varphi}$. Because $(p \varphi(s)+q)(\varphi(s)+s)$ is a non-zero rational function, the section $\sigma$ is not of order two, which proves that it is non-torsion.

Proof of (2). Since $\operatorname{deg} f=4$, we can assume without loss of generality that $f(t)=a t^{4}+b t^{2}+c t+d$ for certain $a, b, c, d \in \mathbb{Z}$ with $a \neq 0$. From the assumption, there exists $t_{0} \in \mathbb{Q}$ such that $\left(x_{0}, y_{0}, t_{0}\right)$ is a rational point on $\mathcal{E}_{f}$ and $x_{0} \neq 0$. Then $\left(x_{0}, y_{0} / x_{0}, t_{0}\right)$ is a rational point on $\mathcal{E}_{f}^{\prime}$.

Set $X=p T^{2}+q T+x_{0}, Y=r T+y_{0} / x_{0}, t=T+t_{0}$. For these $X, Y, t$ we get

$$
F(X, Y, t)=\left(a_{1} T+a_{2} T^{2}+a_{3} T^{3}+a_{4} T^{4}\right) / x_{0}^{2}
$$

where

$$
\begin{aligned}
& a_{1}=x_{0}^{2}\left(c+2 b t_{0}+4 a t_{0}^{3}-2 r y_{0}\right)+q\left(2 x_{0}^{3}-y_{0}^{2}\right), \\
& a_{2}=x_{0}\left(b x_{0}+q^{2} x_{0}+6 a t_{0}^{2} x_{0}-r^{2} x_{0}^{2}-2 q r y_{0}\right)+p\left(2 x_{0}^{3}-y_{0}^{2}\right), \\
& a_{3}=x_{0}\left(2 p q x_{0}-q r^{2} x_{0}+4 a t_{0} x_{0}-2 p r y_{0}\right), \\
& a_{4}=\left(a+p^{2}-p r^{2}\right) x_{0}^{2} .
\end{aligned}
$$

If now $2 x_{0}^{3}-y_{0}^{2} \neq 0$, then the system of equations $a_{1}=a_{2}=0$ is triangular with respect to $p, q$. Because the curve $E_{t_{0}}$ has infinitely many rational points, for fixed $a, b, c, d$ we can choose $x_{0}, y_{0}$ such that $2 x_{0}^{3}-y_{0}^{2} \neq 0$, and the system $a_{1}=a_{2}=0$ has a solution satisfying $p \neq 0$ or $q \neq 0$. Therefore, we obtain

$$
\begin{aligned}
& q=-\frac{x_{0}^{2}\left(c+2 b t_{0}+4 a t_{0}^{3}-2 r y_{0}\right)}{2 x_{0}^{3}-y_{0}^{2}}, \\
& p=-\frac{x_{0}\left(b x_{0}+q^{2} x_{0}+6 a t_{0}^{2} x_{0}-r^{2} x_{0}^{2}-2 q r y_{0}\right)}{2 x_{0}^{3}-y_{0}^{2}} .
\end{aligned}
$$


For these $p, q$, the equation $F\left(p T^{2}+q T+x_{0}, r T+y_{0} / x_{0}, T+t_{0}\right)=0$ has a triple root $T=0$ and another root

$$
T=-\frac{2 p q x_{0}-q r^{2} x_{0}+4 a t_{0} x_{0}-2 p r y_{0}}{\left(a+p^{2}-p r^{2}\right) x_{0}}=: \psi(r)-t_{0} .
$$

If we now put $t=\psi(r)$, then the surface $\mathcal{E}_{f \circ \psi}$ has a section

$$
\sigma=\left(p T^{2}+q T+x_{0},\left(r T+y_{0} / x_{0}\right)\left(p T^{2}+q T+x_{0}\right)\right),
$$

where $p, q$ are given by (2.2) and $T$ is given by (2.3). Since $\left(r T+y_{0} / x_{0}\right)\left(p T^{2}+\right.$ $\left.q T+x_{0}\right) \neq 0$, the section $\sigma$ is not of order two, which proves that it is non-torsion.

Here a natural and non-trivial problem is to construct polynomials $f$ of degree four for which there is a rational point with $y \neq 0$ on the surface $\mathcal{E}_{f}$. It turns out that there exists a wide class of polynomials with this property.

Now we will show the following

Theorem 2.2. If $f \in \mathbb{Q}[t], \operatorname{deg} f=4$ and $f(t) \neq f(-t)$, then there exists a rational base change $t=\varphi(u)$ such that the surface $\mathcal{E}_{\text {fo }}$ has a non-torsion section.

Proof. We can assume that $f(t)=a t^{4}+b t^{2}+c t+d$ for some $a, b, c, d \in \mathbb{Z}$ with $a c \neq 0$. Let $u$ be a variable, put $x=a u^{2}$ and treat our surface as a curve of degree 4 defined over $\mathbb{Q}(u)$, i.e. we consider the curve

$$
C_{1}: y^{2}=a^{2} u^{2} t^{4}+a b u^{2} t^{2}+a c u^{2} t+a d u^{2}+a^{3} u^{6}=: h_{1}(t) .
$$

Note that the point at infinity on $C_{1}$ is rational. Now put $t=T, y=a u T^{2}+$ $p T+q$. Then

$$
\left(a u T^{2}+p T+q\right)^{2}-h_{1}(T)=a_{0}+a_{1} T+a_{2} T^{2}+a_{3} T^{3},
$$

where

$$
\begin{array}{ll}
a_{0}=-q^{2}+a d u^{2}+a^{3} u^{6}, & a_{1}=-2 p q+a c u^{2}, \\
a_{2}=-p^{2}-2 a q u+a b u^{2}, & a_{3}=-2 a p u .
\end{array}
$$

The system $a_{2}=a_{3}=0$ has a solution $p=0, q=b u / 2$. For these $p, q$, the equation $\left(a u T^{2}+p T+q\right)^{2}-h_{1}(T)=0$ has a root

$$
T=-\frac{-b^{2}+4 a d+4 a^{3} u^{4}}{4 a c}=: \varphi(u)
$$

We have shown that if $a c \neq 0$ then the surface $\mathcal{E}_{f \circ \varphi}$ has a section

$$
\sigma_{1}=\left(a u^{2}, \frac{\left(-b^{4}-8 a b c^{2}+8 a b^{2} d-16 a^{2} d^{2}\right) u+8 a^{3}\left(b^{2}-4 a d\right) u^{5}-16 a^{6} u^{9}}{16 a c^{2}}\right),
$$

which is clearly non-torsion.

From the above theorem we obtain two interesting corollaries: 
Corollary 2.3. If $f(t)=a t^{4}+b t^{2}+c t+d \in \mathbb{Z}[t], a, c \in\{-1,1\}$ and $b \equiv 0(\bmod 2)$, then the diophantine equation $y^{2}=x^{3}+f(t) x$ has infinitely many solutions in integers.

Corollary 2.4. If $f \in \mathbb{Q}[t], \operatorname{deg} f=4, f$ is not even and has at least two complex roots, then the diophantine equation $v^{2}=u^{4}+f(w)$ has infinitely many rational parametric solutions.

Proof. Write $\mathcal{S}: v^{2}=u^{4}+f(w)$. The method described in [7, p. 77] shows that $\mathcal{S}$ is birationally equivalent to the surface

$$
\mathcal{E}: y^{2}=x^{3}-4 f(t) x .
$$

The relevant mapping from $\mathcal{S}$ to $\mathcal{E}$ is given by

$$
(u, v, w)=\left(\frac{y}{2 x}, \frac{2 x^{3}+y^{2}}{4 x^{2}}, t\right),
$$

with inverse

$$
(x, y, t)=\left(-2\left(u^{2}-v\right),-4 u\left(u^{2}-v\right), w\right) .
$$

Applying now Theorem 2.2 we obtain the statement of our corollary.

An interesting question is whether there exists a non-trivial rational point on $\mathcal{E}_{f}$ if $f(t)=a t^{4}+b t^{2}+d$ for some $a, b, d \in \mathbb{Z}$. Note that if the equation $f(t)=0$ has a rational root $t_{0}$, then on $\mathcal{E}_{f}$ we have a rational curve $(x, y, t)=$ $\left(u^{2}, u^{3}, t_{0}\right)$ and we can use the second part of Theorem 2.1 to construct other rational curves on $\mathcal{E}_{f}$. Without any difficulty we can give other infinite families of polynomials satisfying condition (2) of Theorem 2.1. For instance, if $f(t)=a t^{4}+b t^{2}+u\left(v^{2}-u\right)$, then on the curve $E_{0}: y^{2}=x^{3}+u\left(v^{2}-u\right) x$ there is a point $(u, u v)$ which is not of finite order if $u v \neq 0$.

One can check by computer that if $\max \{|a|,|b|,|d|\} \leq 100$, then there exists $t \in \mathbb{Q}$ such that there are infinitely many rational points on the curve $E_{t}: y^{2}=x^{3}+f(t) x$. This leads us to the following

CONJECTURE 2.5. Let $a, b, d \in \mathbb{Z}$ and $f(t)=a t^{4}+b t^{2}+d$. Then there exists $t_{0} \in \mathbb{Q}$ such that there are infinitely many rational points on the curve $E_{t_{0}}$.

3. Rational points on $\mathcal{E}^{g}: y^{2}=x^{3}+g(t)$. Let $g \in \mathbb{Q}[t]$ be a monic polynomial of degree 6 and assume $g(t) \neq t^{6}$. For such $g$ consider the surface

$$
\mathcal{E}^{g}: y^{2}=x^{3}+g(t) .
$$

For fixed $t \in \mathbb{Q}$, denote the curve $y^{2}=x^{3}+g(t)$ by $E^{t}$. Its torsion part is as follows ([12, p. 323]). If $g(t)=1$, then Tors $E^{t} \cong \mathbb{Z} / 6 \mathbb{Z}$. If $g(t) \neq 1$ and $g(t)$ is a square in $\mathbb{Q}$, then Tors $E^{t}=\{\mathcal{O},(0, \sqrt{g(t)}),(0,-\sqrt{g(t)})\}$. If $g(t)=-432$ we have Tors $E^{t}=\{\mathcal{O},(12,36),(12,-36)\}$. If $g(t) \neq 1$ and $g(t)$ is a cube in $\mathbb{Q}$, then Tors $E^{t}=\{\mathcal{O},(-\sqrt[3]{g(t)}, 0)\}$. In the remaining cases, Tors $E^{t}=\{\mathcal{O}\}$. 
As an immediate consequence we see that if there is a rational base change $t \mapsto \beta(t)$ such that the surface $\mathcal{E}^{g \circ \beta}$ has a section $\sigma=(x, y)$ with $x y \neq 0$, then $\sigma$ is non-torsion.

We show the following

TheOREM 3.1. Let $g \in \mathbb{Q}[t]$ be a monic polynomial of degree six. If $g$ is not even, then there exists a rational base change $t=\chi(u)$ such that the surface $\mathcal{E}^{g \circ \chi}$ has a non-torsion section.

Proof. We can assume that $g(t)=t^{6}+a t^{4}+b t^{3}+c t^{2}+d t+e$ for some $a, b, c, d, e \in \mathbb{Z}$ with $b \neq 0$ or $d \neq 0$. Let now $C_{2}$ denote the curve over $\mathbb{Q}(t)$ obtained from $\mathcal{E}^{g}$ after substituting $x=\left(u^{2}-a\right) / 3-t^{2}$. Then

$$
\begin{aligned}
C_{2}: y^{2}= & u^{2} t^{4}+b t^{3}-\frac{a^{2}-3 c-2 a u^{2}+u^{4}}{3} t^{2}+d t \\
& +\frac{-a^{3}+27 e+3 a^{2} u^{2}-3 a u^{4}+u^{6}}{27}=: h_{2}(t) .
\end{aligned}
$$

Note that the point at infinity on $C_{2}$ is rational. Set $t=T, y=u T^{2}+p T+q$. Then

$$
\left(u T^{2}+p T+q\right)^{2}-h_{2}(T)=a_{0}+a_{1} T+a_{2} T^{2}+a_{3} T^{3},
$$

where

$$
\begin{array}{ll}
a_{0}=\frac{a^{3}-27 e+27 q^{2}-3 a^{2} u^{2}+3 a u^{4}-u^{6}}{27}, & a_{1}=-d+2 p q, \\
a_{2}=\frac{a^{2}-3 c+3 p^{2}+6 q u-2 a u^{2}+u^{4}}{3}, & a_{3}=-b+2 p u .
\end{array}
$$

Solving the system $a_{2}=a_{3}=0$ with respect to $p, q$ we obtain

$$
p=\frac{b}{2 u}, \quad q=\frac{-3 b^{2}-4 a^{2} u^{2}+12 c u^{2}+8 a u^{4}-4 u^{6}}{24 u^{3}} .
$$

Now, if $p, q$ are given by (3.1), then the equation $\left(u T^{2}+p T+q\right)^{2}-h_{2}(T)=0$ has a root $T=-\chi_{1}(u) / \chi_{2}(u)=: \chi(u)$, where

$$
\begin{aligned}
\chi_{1}(u)= & -27 b^{4}-72 b^{2}\left(a^{2}-3 c\right) u^{2}-48\left(a^{4}-3 a b^{2}-6 a^{2} c+9 c^{2}\right) u^{4} \\
& +8\left(16 a^{3}-9 b^{2}-72 a c+216 e\right) u^{6}-96\left(a^{2}-3 c\right) u^{8}+16 u^{12}, \\
\chi_{2}(u)= & 72 u^{2}\left(3 b^{3}+4 b\left(a^{2}-3 c\right) u^{2}-8(a b-3 d) u^{4}+4 b u^{6}\right) .
\end{aligned}
$$

Our computations imply that the surface $\mathcal{E}^{g \circ \chi}$ has a section $\sigma_{2}=\left(\left(u^{2}-a\right.\right.$ $\left.\left.-3 T^{2}\right) / 3, u T^{2}+p T+q\right)$, where $p, q$ are given by $(3.1)$ and $T=\chi(u)$. It is easy to see that $\sigma_{2}$ is non-torsion.

Note that the assumption " $b \neq 0$ or $d \neq 0$ " is essential for the method employed because in the opposite case the function $\chi_{2}$ is identically zero.

Here a natural question arises whether the assumption that for some $t_{0} \in \mathbb{Q}$ there are infinitely many rational points on the curve $E^{t_{0}}$ enables one to construct a rational curve on the surface $\mathcal{E}^{g}$. Unfortunately, we are not 
able to give such a construction for any even polynomial $g$. However, we can prove

THEOREM 3.2. Let $g \in \mathbb{Q}[t]$ be a monic and even polynomial of degree six. If there exists $t_{0} \in \mathbb{Q}$ such that there are infinitely many rational points on the curve $E^{t_{0}}$, then the set of $t \in \mathbb{Q}$ such that $E^{t}$ has positive rank is infinite.

Proof. Because $g$ is even we can assume that $g(t)=t^{6}+a t^{4}+c t^{2}+e$ for some $a, c, e \in \mathbb{Z}$ with $a \neq 0$ or $c \neq 0$. The case $a=c=0$ will be discussed in the next section. For the proof it will be convenient to work with the surface $\mathcal{F}^{g}$ given by the equation

$$
\mathcal{F}^{g}: Y^{2}+2 t^{3} Y=X^{3}+a t^{4}+c t^{2}+e .
$$

Set $G(X, Y, t):=Y^{2}+2 t^{3} Y-\left(X^{3}+a t^{4}+c t^{2}+e\right)$. Then $\mathcal{E}^{g}$ is birationally equivalent to $\mathcal{F}^{g}$ via the mapping $(x, y, t)=\left(X, Y+t^{3}, t\right)$ with inverse $(X, Y, t)=\left(x, y-t^{3}, t\right)$. By assumption there exists $t_{0} \in \mathbb{Q}$ such that there are infinitely many rational points on $E^{t_{0}}$. Thus, there is a rational point $\left(x_{0}, y_{0}, t_{0}\right)$ on $\mathcal{E}^{g}$ such that $x_{0} y_{0} \neq 0$. Then $\left(x_{0}, y_{0}-t_{0}^{3}, t_{0}\right)$ is on $\mathcal{F}^{g}$. Set $X=p T+x_{0}, Y=q T+y_{0}-t_{0}^{3}, t=T+t_{0}$. Then

$$
G(X, Y, t)=a_{1} T+a_{2} T^{2}+a_{3} T^{3}+a_{4} T^{4},
$$

where

$$
\begin{aligned}
& a_{1}=-3 p x_{0}^{2}+2 q y_{0}-2 c t_{0}-4 a t_{0}^{3}-6 t_{0}^{5}+6 t_{0}^{2} y_{0}, \\
& a_{2}=q^{2}+6 q t_{0}^{2}-3 p^{2} x_{0}-c+6 a t_{0}^{2}-6 t_{0}^{4}+6 t_{0} y_{0}, \\
& a_{3}=-p^{3}+6 q t_{0}-4 a t_{0}-2 t_{0}^{3}+2 y_{0}, \\
& a_{4}=2 q-a .
\end{aligned}
$$

Solving the system $a_{1}=a_{4}=0$ with respect to $p, q$ we obtain

$$
p=-\frac{2 c t_{0}+4 a t_{0}^{3}+6 t_{0}^{5}-a y_{0}-6 t_{0}^{2} y_{0}}{3 x_{0}^{2}}, \quad q=\frac{a}{2} .
$$

For these $p, q$, the equation $G\left(p T+x_{0}, q T+y_{0}-t_{0}^{3}, T+t_{0}\right)=0$ has a root $T=0$ and another root

$$
T=-\frac{q^{2}+6 q t_{0}^{2}-3 p^{2} x_{0}-c+6 a t_{0}^{2}-6 t_{0}^{4}+6 t_{0} y_{0}}{-p^{3}+6 q t_{0}-4 a t_{0}-2 t_{0}^{3}+2 y_{0}} .
$$

From the above computations we see that the point $\left(p T+x_{0}, q T+y_{0}-t_{0}^{3}\right.$, $\left.T+t_{0}\right)$, for $p, q$ given by (3.2) and $T$ defined by (3.3), lies on $\mathcal{F}^{g}$. Hence the point $P=\left(p T+x_{0}, q T+y_{0}-t_{0}^{3}+\left(T+t_{0}\right)^{3}, T+t_{0}\right)$ is on $\mathcal{E}^{g}$. Because the set of rational points on $E^{t_{0}}$ is infinite, we can assume that the coordinates of $P$ are non-zero, $g(T) \neq 0,-432$ and $g\left(T+t_{0}\right) / g\left(t_{0}\right)$ is not a sixth power. If we now put $t_{1}=T+t_{0}$, then the curve $E^{t_{1}}$ has infinitely many rational points. 
Now suppose that we have already constructed $t_{1}, \ldots, t_{n}$ such that $E^{t_{i}}$ has a positive rank for $i=1, \ldots, n$. Then we can apply the above procedure to the point $\left(x_{n}, y_{n}, t_{n}\right)$, where $\left(x_{n}, y_{n}\right)$ is a rational point on $E^{t_{n}}$ such that $T$ given by (3.3) satisfies: $g\left(T+t_{n}\right) \neq 0,-432$ and $g\left(T+t_{n}\right) / g\left(t_{i}\right)$ is not a sixth power for $i=1, \ldots, n$. Why can we find such a $T$ ? If $g$ has no root of multiplicity 5 , then there are only finitely many rational points on every curve $g(u)=g\left(t_{i}\right) v^{6}$ (of genus $>1$ ) for $i=1, \ldots, n$. This is an immediate consequence of the Faltings theorem [3]. The case when $g$ has a root of multiplicity 5 (it is then a rational root) can be easily excluded, as then the surface $\mathcal{E}^{g}$ is rational over $\mathbb{Q}$. Because there are infinitely many rational points on the curve $E^{t_{n}}$, we see that $T$ given by (3.3) can be selected to satisfy all the necessary conditions. Now using the previous reasoning we can construct infinitely many $t \in \mathbb{Q}$ such that $E^{t}$ has a positive rank.

REMARK 3.3. Note that if $g\left(t_{0}\right)=0$ for a rational number $t_{0}$, then the set of rational points on the curve $E^{t_{0}}: y^{2}=x^{3}$ is parametrized by $x=u^{2}, y=u^{3}$. Using the reasoning from Theorem 3.2 we can easily deduce that in this case it is possible to construct a rational curve on $\mathcal{E}^{g}$.

From the above remark we obtain the following

Corollary 3.4. Let $h \in \mathbb{Q}[t]$ with $\operatorname{deg} h=5, h(0)=1$ and consider the surface $\mathcal{S}: y^{2}=x^{3}+h(t)$. Then there is a rational base change $t=\gamma(u)$ such that the surface $\mathcal{S}^{\gamma}: y^{2}=x^{3}+h(\gamma(u))$ has a non-torsion section.

Proof. Note that $\mathcal{S}$ is birationally equivalent to $\mathcal{E}^{g}$, where $g(t)=t^{6} h(1 / t)$. The mapping from $\mathcal{S}$ to $\mathcal{E}^{g}$ is given by $(x, y, t) \mapsto\left(x / t^{2}, y / t^{3}, 1 / t\right)$. Because $g(0)=0$, we can use Remark 3.3 to obtain the statement.

EXAmple 3.5. Let $g(t)=t^{6}+t^{2}+1$ and consider the surface $\mathcal{E}^{g}$ : $y^{2}=x^{3}+g(t)$. For $t_{0}=1$, on the curve $E^{1}: y^{2}=x^{3}+3$ we have a non-torsion point $P=(1,2)$. Now we calculate the quantities $p, q$ given by (3.2) and $T$ given by (3.3) from the proof of Theorem 3.2. We obtain $p=16 / 13, q=-1 / 13, T=-358 / 169$ and next $t_{1}=T+t_{0}=-189 / 169$. Thus, on the curve

$$
E^{t_{1}}: y^{2}=x^{3}+\frac{47 \cdot 2085456070589}{13^{12}}
$$

we have a non-torsion point

$$
P=\left(-\frac{3531}{2197}, \frac{1137934}{4826809}\right)
$$

Similarly to the case of the surface $\mathcal{E}_{f}$ considered in Section 2, we can ask whether for a given polynomial $g$ of the form $g(t)=t^{6}+a t^{4}+c t^{2}+e$ there is $t_{0} \in \mathbb{Q}$ such that the curve $E^{t_{0}}$ has infinitely many rational points. 
In the following section we will prove that the answer is positive for polynomials of the form $g(t)=t^{6}+e$. We checked by computer that if $\max \{|a|,|c|,|e|\} \leq 10$, then there exists $t \in \mathbb{Q}$ such that there are infinitely many rational points on the curve $E^{t}: y^{2}=x^{3}+t^{6}+a t^{4}+c t^{2}+e$. This leads us to the following

Conjecture 3.6. Let $a, c, e \in \mathbb{Z}$ and $g(t)=t^{6}+a t^{4}+c t^{2}+e$. Then there exists $t_{0} \in \mathbb{Q}$ such that there are infinitely many rational points on the curve $E^{t_{0}}$.

In view of Theorem 3.2 a natural question arises:

QUESTION 3.7. Let $g(t)=t^{6}+a t^{4}+c t^{2}+e$. What conditions guarantee the existence of a rational base change $t=\kappa(u)$ such that the surface $\mathcal{E}^{g \circ \kappa}$ has a non-torsion section?

4. Some results on the diophantine equation $x^{2}-y^{3}-g(z)=t$. Let $g(z)=z^{6}+a z^{4}+b z^{3}+c z^{2}+d z+e \in \mathbb{Z}[z]$ and let $t$ be a variable. In this section we will deal with the diophantine equation

$$
x^{2}-y^{3}-g(z)=t .
$$

We will show that if there are infinitely many rational points on the curve $C: v^{2}=s^{4}-12 a s^{2}+48 b s+6\left(a^{2}-12 c\right)$, then the equation (4.1) has infinitely many solutions in $\mathbb{Q}[t]$. For $g(t)=t^{6}+e$, we will use this result to prove the promised theorem on the existence of a rational base change $t=\chi_{1}(s)$ such that there exists a non-torsion section on $\mathcal{E}^{g \circ \chi_{1}}$. We will also give some results concerning the representability of integers in the form $x^{2}-y^{3}-g(z)$.

We start with the following

THEOREM 4.1. If there are infinitely many rational points on the curve $C: v^{2}=s^{4}-12 a s^{2}+48 b s+6\left(a^{2}-12 c\right)$, in particular if $b \neq 0$ and $a \equiv 1$ $(\bmod 2)$, then the equation $(4.1)$ has infinitely many solutions in $\mathbb{Q}[t]$.

Proof. Set $G(x, y, z):=x^{2}-y^{3}-g(z)$ and observe that solving the equation $G(x, y, z)=t$ in polynomials with rational coefficients is equivalent to constructing polynomials $x, y, z \in \mathbb{Q}[t]$ such that $\operatorname{deg} G(x(t), y(t), z(t))=1$. Now put $x=3 T^{3}+p T^{2}+q T+r, y=2 T^{2}+s T+u, z=T$. Then

$$
G\left(3 T^{3}+p T^{2}+q T+r, 2 T^{2}+s T+u, T\right)=a_{0}+a_{1} T+a_{2} T^{2}+a_{3} T^{3}+a_{4} T^{4}+a_{5} T^{5},
$$

where

$$
\begin{array}{ll}
a_{0}=r^{2}-u^{3}-e, & a_{1}=-d+2 q r-3 s u^{2}, \\
a_{2}=-c+q^{2}+2 p r-3 s^{2} u-6 u^{2}, & a_{3}=-b+2 p q+6 r-s^{3}-12 s u, \\
a_{4}=-a+p^{2}+6 q-6 s^{2}-12 u, & a_{5}=6(p-2 s) .
\end{array}
$$


Solving the system $a_{3}=a_{4}=a_{5}=0$ with respect to $p, q, r$ we obtain

$$
p=2 s, \quad q=\frac{a+2 s^{2}+12 u}{6}, \quad r=\frac{3 b-2 a s-s^{3}+12 s u}{18} .
$$

After substituting $p, q, r$ into the equation $a_{2}=0$ and solving it with respect to $u$, we obtain

$$
u=\frac{3 s^{2}+2 a \pm \sqrt{s^{4}-12 a s^{2}+48 b s+6\left(a^{2}-12 c\right)}}{12} .
$$

Thus, we can see that if there are infinitely many rational points on the curve

$$
C: v^{2}=s^{4}-12 a s^{2}+48 b s+6\left(a^{2}-12 c\right)=: U(s),
$$

then all but finitely many points on $C$, by (4.2) and (4.3), give us a triple of polynomials $x, y, z \in \mathbb{Q}[T]$ such that $G(x(T), y(T), z(T))=a_{1} T+a_{0}$ and $a_{1} \neq 0$. After substitution $T=\left(t-a_{0}\right) / a_{1}$ we obtain a solution of the equation $x^{2}-y^{3}-g(z)=t$. Moreover, note that we always have infinitely many rational points on $C$ when the polynomial $U$ has multiple roots, which is equivalent to the condition $D:=25 a^{6}-144 a^{3} b^{2}-2592 b^{4}-180 a^{4} c+$ $5184 a b^{2} c-1296 a^{2} c^{2}-1728 c^{3}=0$.

Since the curve $C$ is rational over $\mathbb{Q}$ if $D=0$, we can assume that $D \neq 0$. To show that if $b \neq 0$ and $a \equiv 1(\bmod 2)$, then there are infinitely many rational points on $C$, we transform $C$ into an elliptic curve with the Weierstrass equation. We can do this because the point at infinity on $C$ is rational. Using the method described in [7] one more time, we birationally transform $C$ into the curve

$$
E: Y^{2}=X^{3}-72\left(a^{2}-4 c\right) X+64\left(a^{3}+36 b^{2}-36 a c\right) \text {. }
$$

The mapping transforming $C$ into $E$ is

$$
(s, v)=\left(\frac{48 b-Y}{16 a-2 X}, 2 a+\frac{X}{2}-\left(\frac{48 b-Y}{16 a-2 X}\right)^{2}\right),
$$

with inverse

$$
(X, Y)=\left(2\left(-2 a+s^{2}+v\right), 4\left(12 b-6 a s+s^{3}+s v\right)\right) .
$$

Note that the rational point $P=(8 a, 48 b)$ lies on the curve $E$. Using the chord and tangent method of adding points on an elliptic curve, we obtain $2 P=\left(x_{1}, y_{1}\right)$ where

$$
\begin{aligned}
& x_{1}=\frac{25 a^{4}-256 a b^{2}+120 a^{2} c+144 c}{16 b^{2}}, \\
& y_{1}=48 b+\frac{\left(5 a^{2}+12 c\right)\left(25 a^{4}-384 a b^{2}+120 a^{2} c+144 c^{2}\right)}{64 b^{3}} .
\end{aligned}
$$

Because $a \equiv 1(\bmod 2)$ the numerator of $x_{1}$ is odd, so $x_{1} \in \mathbb{Q} \backslash \mathbb{Z}$. By the Nagell-Lutz theorem ([12, p. 77]) torsion points on an elliptic curve $y^{2}=x^{3}+p x+q, p, q \in \mathbb{Z}$, have integer coordinates, so the point $2 P$ is 
not of finite order. This proves that the curve $E$ has a positive rank and we conclude that there are infinitely many rational points on the curve $C$.

REMARK 4.2. After noticing that the point $P=(8 a, 48 b)$ lies on the curve $E$ (see the proof above), we suspected that this point is not of finite order for $a b \neq 0$ and any $c \in \mathbb{Z}$. As suggested by Professor Schinzel, this is not true. Indeed, if $a=6 p^{2}, c=p\left(4 b-15 p^{3}\right)$, then the curve $E$ is elliptic if $\Delta=-764411904 b^{2}\left(3 b-16 p^{3}\right) \neq 0$. In this case the point $P=\left(6 p^{2}, 48 b\right)$ is of order three on $E$. If we now put $p=1, b=1$, then $a=6, c=-11$. The curve $E$ is then birationally equivalent to $E^{\prime}: y^{2}=x^{3}-360 x+2628$. Applying APECS [2] we found that the rank of $E^{\prime}$ is zero. Despite this, there exists a non-trivial solution of the equation $x^{2}-y^{3}-g(z)=t$ for $b=1$ and even for $b \neq 0$; this is equivalent to the fact that the point $P$ is not of order two. Why is it so? If $b \neq 0$, then the order of $P$ is at least 3 and the $s$-coordinate of the preimage of $2 P$ (different from the point at infinity $\mathcal{O}$ ) equals $\left(5 a^{2}+12 c\right) / 18 b$. Because the expression $a_{1}$ from the proof of Theorem 4.1 depends linearly on $d$ and is not identically zero, there is at least one $d \in \mathbb{Z}$ for which $a_{1}=0$ and our method does not give a solution of the equation $x^{2}-y^{3}-g(z)=t$.

It should be noted that there exists a polynomial $g \in \mathbb{Z}[t]$ for which our method does not give a solution of the equation $x^{2}-y^{3}-g(z)=t$. For example, if $g(t)=t^{6}+6 t^{4}+6 t^{3}+9 t^{2}-150 t$, then the curve $C$ is birationally equivalent to the elliptic curve $E^{\prime}: y^{2}+y=x^{3}-7$. We have Tors $E^{\prime}=\{\mathcal{O},(3,4),(3,-5)\}$ and using APECS once again, we find that $E^{\prime}$ has rank zero. In this case, our method leads to the identity

$$
\left(3 T^{3}+12 T^{2}+33 T+25\right)^{2}-\left(2 T^{2}+6 T+10\right)^{3}-g(T)=-375 .
$$

Now we record several interesting corollaries of Theorem 4.1.

COROLLARY 4.3. If there are infinitely many rational points on the curve $C: v^{2}=s^{4}-12 a s^{2}+48 b s+6\left(a^{2}-12 c\right)$, then every polynomial $h \in \mathbb{Q}[t]$ can be represented in infinitely many ways in the form $x^{2}-y^{3}-g(z)$, where $x, y, z \in \mathbb{Q}[t]$.

In the following corollary we give the promised proof of the existence of rational curves on the surface $y^{2}=x^{3}+t^{6}+e$.

Corollary 4.4. Let $\mathcal{E}^{g}: y^{2}=x^{3}+g(t)$, where $g(t)=t^{6}+e$. Then there exists a rational base change $t=\chi_{1}(s)$ such that the surface $\mathcal{E}^{g \circ \chi_{1}}: y^{2}=$ $x^{3}+g\left(\chi_{1}(s)\right)$ has a non-torsion section.

Proof. Note that if $a=b=c=0$, then the curve $C$ is rational and the system $a_{2}=a_{3}=a_{4}=a_{5}=0$ from the proof of Theorem 4.1 has exactly two solutions given by

$$
\begin{array}{llll}
p_{1}=2 s, & q_{1}=2 s^{2} / 3, & r_{1}=s^{3} / 18, & u_{1}=s^{2} / 6, \\
p_{2}=2 s, & q_{2}=s^{2}, & r_{2}=s^{3} / 6, & u_{2}=s^{2} / 3 .
\end{array}
$$


For such $p_{i}, q_{i}, r_{i}, u_{i}(i=1,2)$ we obtain the following identities:

$$
\begin{array}{r}
\left(3 T^{3}+2 s T^{2}+\frac{2 s^{2}}{3} T+\frac{s^{3}}{18}\right)^{2}-\left(2 T^{2}+s T+\frac{s^{2}}{6}\right)^{3}-\left(T^{6}+d T+e\right) \\
=-\frac{648 e+s^{6}}{648}-\frac{648 d+6 s^{5}}{648} T \\
\left(3 T^{3}+2 s T^{2}+2 s^{2} T+\frac{s^{3}}{6}\right)^{2}-\left(2 T^{2}+s T+\frac{s^{2}}{3}\right)^{3}-\left(T^{6}+d T+e\right) \\
=-\frac{108 e+s^{6}}{108}-d T
\end{array}
$$

If now $d=0$ and if we put $T=\chi_{1}(s)=-\left(648 e+s^{6}\right) /\left(6 s^{5}\right)$ then the right side of the identity (4.4) disappears and the surface $\mathcal{E}^{g \circ \chi_{1}}: y^{2}=x^{3}+g\left(\chi_{1}(s)\right)$ has a section

$$
\begin{aligned}
\sigma=\left(\frac{419904 e^{2}-648 e s^{6}+s^{12}}{18 s^{10}}\right. & \\
& \left.\quad-\frac{272097792 e^{3}-419904 e^{2} s^{6}+1944 e s^{12}+s^{18}}{72 s^{15}}\right) .
\end{aligned}
$$

It is easy to see that the order of $\sigma$ is not finite.

Recall that $a_{1}=-d+2 q r-3 s u^{2}$, where $q, r, s, u$ are given by (4.2) and (4.3) from the proof of Theorem 4.1.

Corollary 4.5. Let $d \in \mathbb{Z}$ and suppose that on the curve $C: v^{2}=$ $s^{4}-12 a s^{2}+48 b s+6\left(a^{2}-12 c\right)$, there is a rational point which leads to $a_{1} \neq 0$. Then for every integer $n$ the diophantine equation $x^{2}-y^{3}-g(z)=n$ has a solution in rationals $x, y, z$ such that there exists an integer $L_{g}$, depending only on the polynomial $g$, with $L_{g} x, L_{g} y, L_{g} z \in \mathbb{Z}$. In particular, for $g(z)=z^{6}$ we have $L_{g}=124416=2^{9} \cdot 3^{5}$.

Proof. In view of Theorem 4.1 the first part of the statement is obvious. Now putting $d=e=0, s=6$ and next $T=(n+72) / 72$ into the identity (4.4) we obtain

$$
\left(\frac{n^{3}-72 n^{2}+15552 n+373248}{124416}\right)^{2}-\left(\frac{n^{2}-72 n+5184}{2592}\right)^{3}-\left(\frac{n+72}{72}\right)^{6}=n
$$

This proves the second part of the corollary.

COROLlary 4.6. Let $g(z)=z^{6}+d z$. If $d=1$, then for every integer $n$ the diophantine equation $x^{2}-y^{3}-g(z)=n$ has infinitely many solutions in integers. If $d=-72 t^{5}+1$ for some integer $t$, then for every integer $n$ the diophantine equation $x^{2}-y^{3}-g(z)=n$ has a solution in integers.

Proof. Let $n$ be a fixed integer. If $d=-1$, then for the proof we will use the identity (4.5). Set $e=0, s=6 t$ and $T=-432 t^{6}-n$. Then the 
polynomials

$$
\begin{aligned}
x(t)= & 3 n^{3}+12 t\left(-1+324 t^{5}\right) n^{2}+36 t^{2}\left(1-288 t^{5}+46656 t^{10}\right) n \\
& +36 t^{3}\left(-1+432 t^{5}-62208 t^{10}+6718464 t^{15}\right), \\
y(t)= & 2 n^{2}+6 t\left(-1+288 t^{5}\right) n+12 t^{2}\left(1-216 t^{5}+31104 t^{10}\right), \\
z(t)= & -n-432 t^{6}
\end{aligned}
$$

satisfy $x(t)^{2}-y(t)^{3}-g(z(t))=n$.

If now $d=-72 t^{5}-1$, then we put $e=0, s=6 t, T=-n-72 t^{6}$ into the identity (4.4). We find that $x^{2}-y^{3}-g(z)=n$ for

$$
\begin{aligned}
x= & 3 n^{3}+12 t\left(-1+54 t^{5}\right) n^{2}+24 t^{2}\left(1-72^{5}+1944 t^{10}\right) n \\
& +12 t^{3}\left(-1+144 t^{5}-5184 t^{10}+93312 t^{15}\right), \\
y= & 2 n^{2}+6 t\left(-1+48 t^{5}\right) n+6 t^{2}\left(1-72 t^{5}+1728 t^{10}\right), \\
z= & -n-72 t^{6} .
\end{aligned}
$$

5. Rational points on some non-isotrivial elliptic surfaces. In view of our considerations it is natural to ask whether it is possible to obtain similar results for non-isotrivial elliptic surfaces of the form

$$
\mathcal{E}: y^{2}=x^{3}+A(t) x+B(t),
$$

where $A, B \in \mathbb{Q}[t] \backslash\{0\}$. If $t \mapsto \alpha(t)$ is a rational base change, then let $\mathcal{E}_{\alpha}$ denote the surface $\mathcal{E}_{\alpha}: y^{2}=x^{3}+A(\alpha(t)) x+B(\alpha(t))$. Moreover, recall that if $\mathcal{C}: y^{2}=x^{3}+m(t) x+n(t)$, where $m, n \in \mathbb{Z}[t]$, is an elliptic curve over $\mathbb{Q}(t)$, then points of finite order on $\mathcal{C}$ have coordinates in $\mathbb{Z}[t]$.

In this section we will prove a generalization of Theorems 2.1 and 2.2.

Theorem 5.1. Let $\mathcal{E}: y^{2}=x^{3}+f_{4}(t) x+g_{4}(t)$, where $f_{4}, g_{4} \in \mathbb{Q}[t]$. If either $\operatorname{deg} f_{4}=3$ and $\operatorname{deg} g_{4} \leq 4$, or $\operatorname{deg} f_{4}=4$, $\operatorname{deg} g_{4} \leq 4$, and if at least one of the polynomials $f_{4}, g_{4}$ is not even, then there exists a rational base change $t=\psi(s)$ such that the surface $\mathcal{E}_{\psi}$ has a non-torsion section.

Proof. Set $H(x, y, t):=y^{2}-\left(x^{3}+f_{4}(t) x+g_{4}(t)\right)$. First, consider the case when $\operatorname{deg} f_{4}=3$ and $\operatorname{deg} g_{4} \leq 4$. We can assume that $f_{4}(t)=a t^{3}+b t+c$, $g_{4}(t)=d t^{4}+e t^{3}+f t^{2}+g t+h$ for some $a, b, \ldots, h \in \mathbb{Z}$ with $a \neq 0$ and $g_{4}(t) \neq 0$. Set $x=p T+q, y=r T^{2}+s T+u, t=T$. For these $x, y, t$ we obtain

$$
H(x, y, t)=a_{0}+a_{1} T+a_{2} T^{2}+a_{3} T^{3}+a_{4} T^{4},
$$

where

$$
\begin{array}{ll}
a_{0}=-h-c q-q^{3}+u^{2}, & a_{1}=-g-c p-b q-3 p q^{2}+2 s u, \\
a_{2}=-f-b p-3 p^{2} q+s^{2}+2 r u, & a_{3}=-e-p^{3}-a q+2 r s, \\
a_{4}=-d-a p+r^{2} &
\end{array}
$$


The system $a_{2}=a_{3}=a_{4}=0$ has a solution

$$
\begin{aligned}
& p=\frac{-d+r^{2}}{a}, \quad q=-\frac{-d^{3}+a^{3} e+3 d^{2} r^{2}-3 d r^{4}+r^{6}-2 a^{3} r s}{a^{4}}, \\
& u=-\frac{-f-b p-3 p^{2} q+s^{2}}{2 r} .
\end{aligned}
$$

If $p, q, u$ are given by (5.1), then the equation $H\left(p T+q, r T^{2}+s T+u, T\right)=0$ has a solution

$$
T=-\frac{h+c q+q^{3}-u^{2}}{g+c p+b q+3 p q^{2}-2 s u}=: \psi(r, s) .
$$

In this case we obtain a two-parameter solution of the equation defining the surface $\mathcal{E}$. For convenience let $r=1$ and $\psi(s):=\psi(1, s)$. We see that if $p, q, u$ are given by (5.1) and $T=t=\psi(s)$, then the surface $\mathcal{E}_{\psi}$ has a section $\sigma=\left(p T+q, T^{2}+s T+u\right)$. An affine change of variables transforms $\mathcal{E}_{\psi}$ into $\mathcal{E}_{\psi}^{\prime}: y^{2}=x^{3}+f_{4}^{\prime}(s) x+g_{4}^{\prime}(s)$, where $f_{4}^{\prime}, g_{4}^{\prime} \in \mathbb{Z}[s]$. Then $\sigma$ goes to a section $\sigma^{\prime}$ on $\mathcal{E}_{\psi}^{\prime}$. It turns out that the $x$-coordinate of the section $2 \sigma^{\prime}$ belongs to $\mathbb{Q}(s) \backslash \mathbb{Q}[s]$. By the remark at the beginning of this section, $\sigma^{\prime}$ is not of finite order. We omit the lengthy computations of this proof, which are troublesome to perform without computer.

Let us now consider the case when $\operatorname{deg} f_{4}=4, \operatorname{deg} g_{4} \leq 4$ and at least one of $f_{4}, g_{4}$ is not even. We can assume that $f_{4}(t)=a t^{4}+b t^{2}+c t+d, g_{4}(t)=$ $e t^{4}+f t^{3}+g t^{2}+h t+i$, where $a, b, \ldots, i \in \mathbb{Z}, a \neq 0$ and at least one of the numbers $c, f, h$ is not zero. Let now $C_{3}$ denote the curve over $\mathbb{Q}(u)$ obtained from $\mathcal{E}$ after substitution $x=\left(u^{2}-e\right) / a$. Hence,

$$
\begin{aligned}
C_{3}: y^{2}= & u^{2} t^{4}+f t^{3}+\frac{-b e+a g-b u^{2}}{a} t^{2}+\frac{-c e+a h-c u^{2}}{a} t \\
& +\frac{-a^{2} d e-e^{3}+a^{3} i+\left(a^{2} d+3 e^{2}\right) u^{2}-3 e u^{4}+u^{6}}{a^{3}}=: V(t) .
\end{aligned}
$$

Now putting $y=u T^{2}+p T+q, t=T$ we obtain

$$
\left(u T^{2}+p T+q\right)^{2}-V(T)=a_{0}+a_{1} T+a_{2} T^{2}+a_{3} T^{3},
$$

where

$$
\begin{aligned}
& a_{0}=\frac{-a^{2} d e-e^{3}+a^{3} i+\left(a^{2} d+3 e^{2}\right) u^{2}-3 e u^{4}+u^{6}}{a^{3}}, \\
& a_{1}=\frac{-c e+a h-2 a p q+c u^{2}}{a}, \\
& a_{2}=\frac{-b e+a g-a p^{2}-2 a q u+b u^{2}}{a}, \quad a_{3}=f-2 p u .
\end{aligned}
$$


The system $a_{2}=a_{3}=0$ has a solution

$$
p=\frac{f}{2 u}, \quad q=\frac{-a f^{2}-4 b e u^{2}+4 a g u^{2}+4 b u^{4}}{8 a u^{3}} .
$$

For these $p, q$ the equation $\left(u T^{2}+p T+q\right)^{2}-V(T)=0$ has exactly one solution

$$
T=\frac{-a^{2} d e-e^{3}+a^{3} i+\left(a^{2} d+3 e^{2}\right) u^{2}-3 e u^{4}+u^{6}}{a^{2}\left(-c e+a h-2 a p q+c u^{2}\right)}=: \psi_{1}(u) .
$$

Now, putting $t=\psi_{1}(u)$ we obtain the section $\sigma_{1}=\left(\left(u^{2}-e\right) / a, u T^{2}+p T+q\right)$ on the surface $\mathcal{E}_{\psi_{1}}$, and similarly to the previous case, we show that the order of $\sigma_{1}$ is not finite.

Remark 5.2. Let now $K$ be a real field for which $[K: \mathbb{Q}]<\infty$. Consider the elliptic surface $\mathcal{E}: y^{2}=x^{3}+C_{1}(t) x+C_{2}(t)$, where $C_{1}, C_{2} \in \mathbb{Q}[t]$ and $\max \left\{\operatorname{deg} C_{1}, \operatorname{deg} C_{2}\right\}=3$, defined over $K$. Let $S:=\left\{t \in K: \operatorname{rank}\left(\mathcal{E}_{t}\right)>0\right\}$. R. Munshi in [8] has shown that there exists a constant $M$, depending only on $[K: \mathbb{Q}]$, such that if $|S|>M$, then the set $S$ is dense in $\mathbb{R}$ (Theorem 1.9 in [8]). The first part of Theorem 5.1 shows that in fact the surface $\mathcal{E}$ is unirational. Without any additional assumptions this proves that the set $S$ is dense in $\mathbb{R}$.

With a similar method we can prove the following

THEOREM 5.3.

(1) Let $\mathcal{E}: y^{2}=x\left(x^{2}+f_{2}(t) x+f_{4}(t)\right)$, where $f_{2}, f_{4} \in \mathbb{Q}[t]$. If either $\operatorname{deg} f_{2} \leq 2, \operatorname{deg} f_{4} \leq 3$, or $\operatorname{deg} f_{2} \leq 2, \operatorname{deg} f_{4}=4$, and if at least one of the polynomials $f_{2}, f_{4}$ is not even, then there exists a rational base change $t=\psi(u)$ such that the surface $\mathcal{E}_{\psi}$ has a non-torsion section.

(2) If $\operatorname{deg} f_{2}=2, \operatorname{deg} f_{4}=4$ and there is $t_{0} \in \mathbb{Q}$ such that the curve $\mathcal{E}_{t_{0}}: y^{2}=x\left(x^{2}+f_{2}\left(t_{0}\right) x+f_{4}\left(t_{0}\right)\right)$ has infinitely many rational points, then there exists a rational base change $t=\psi(u)$ such that $\mathcal{E}_{\psi}$ has a non-torsion section.

(3) Let $\mathcal{E}: y^{2}=x\left(x^{2}+f_{4}(t) x+g_{4}(t)\right)$, where $f_{4}, g_{4} \in \mathbb{Q}[t]$ and $\operatorname{deg} f_{4}=$ $\operatorname{deg} g_{4}=4$. If $f_{4}$ or $g_{4}$ is not even, then there exists a rational base change $t=\psi(u)$ such that $\mathcal{E}_{\psi}$ has a non-torsion section.

Proof. The proofs of (1) and (2) do not bring any difficulties, and therefore they will be omitted (the reasoning is exactly the same as in the proof of Theorem 2.1).

We now outline the proof of (3). Let $a, b$ be the leading coefficients of $f_{4}, g_{4}$, respectively. Set $x=b /\left(u^{2}-a\right)$ and treat $\mathcal{E}$ as a curve defined over $\mathbb{Q}(u)$; denote the latter curve by $C_{4}$. Then the point at infinity, say $P$, on $C_{4}$ is rational. Since $f_{4}$ or $g_{4}$ is not even, with the use of the point $P$ we can construct a non-torsion section on $\mathcal{E}$. 
The previously considered elliptic surfaces (excluding the one from Theorem 5.3(3)) are rational over $\mathbb{C}$. This means that they are rational over a certain finite extension of $\mathbb{Q}$. Can one say something more about rational points on general elliptic surfaces? With reference to this question, in 1992 B. Mazur proposed an interesting conjecture concerning rational points on $\mathcal{E}$. For $t \in \mathbb{Q}$ let $\mathcal{E}_{t}$ be the fibre of the mapping $\pi: \mathcal{E} \rightarrow \mathbb{P}$ over $t$.

Conjecture 5.4 (Conjecture 4 from [6]). The family $\left\{\mathcal{E}_{t}\right\}_{t \in \mathbb{Q}}$ of elliptic curves satisfies one of the following conditions:

(1) for all but finitely many $t \in \mathbb{Q}$ the curve $\mathcal{E}_{t}$ has Mordell-Weil rank zero,

(2) there exists a set $R \subset \mathbb{Q}$ such that $R$ is dense in $\mathbb{R}$ and for each $t \in R$, the Mordell-Weil rank of $\mathcal{E}_{t}$ is positive.

As pointed out in [6], the only known example of an elliptic surface whose fibres satisfy condition (1) of the above conjecture is the split elliptic surface $\mathcal{E} \simeq E \times \mathbb{C}$, where $E$ is an elliptic curve of rank zero. It seems plausible that if the family $\left\{\mathcal{E}_{t}\right\}_{t \in \mathbb{Q}}$ comes from a non-split elliptic surface, then (1) is not valid. It should be mentioned that recently N. Elkies gave heuristic arguments which suggest that certain isotrivial families of elliptic curves over $\mathbb{Q}$ violate Mazur's conjectured rule.

Examples of families of elliptic surfaces satisfying condition (2) of the above conjecture can be found in [4], [10], [5], [8].

We believe that the following conjecture may be easier to prove:

CONJECTURE 5.5. Let $\mathcal{E}$ be a non-split elliptic surface. Then there is $t \in \mathbb{Q}$ such that the curve $\mathcal{E}_{t}$ has infinitely many rational points.

As a corollary, we obtain an interesting

Theorem 5.6. Assume that Conjecture 5.5 is true. Then for the family $\left\{\mathcal{E}_{t}\right\}_{t \in \mathbb{Q}}$ of elliptic curves, the set of rational numbers $t$ such that the rank of $\mathcal{E}_{t}$ is positive, is infinite.

Proof. Assuming Conjecture 5.5 to be true, we find $t_{1} \in \mathbb{Q}$ such that there are infinitely many rational points on the curve $\mathcal{E}_{t_{1}}$. Suppose that we have already constructed $t_{2}, \ldots, t_{n}$ such that the curve $\mathcal{E}_{t_{i}}$ for $i=1, \ldots, n$ has infiniteley many rational points. Further suppose that there is a polynomial $h \in \mathbb{Q}[t]$ such that for $i=1, \ldots, n$ the equation $h(t)=t_{i}$ has no solutions in rationals and the system of equations

$$
\left\{\begin{array}{ccc}
A\left(t_{1}\right) Y_{1}^{4}=A\left(h\left(T_{1}\right)\right), & B\left(t_{1}\right) Y_{1}^{6}=B\left(h\left(T_{1}\right)\right), \\
\vdots & \vdots \\
A\left(t_{n}\right) Y_{n}^{4}=A\left(h\left(T_{n}\right)\right), & B\left(t_{n}\right) Y_{n}^{6}=B\left(h\left(T_{n}\right)\right) .
\end{array}\right.
$$


also has no such solutions. Then there exists $t \in \mathbb{Q}$ such that the curve $\mathcal{E}_{h(t)}: y^{2}=x^{3}+A(h(t)) x+B(h(t))$ has a positive rank. Defining now $t_{n+1}=h(t)$ and repeating the reasoning, we obtain the statement of the theorem.

We now show that there exists $h \in \mathbb{Q}[t]$ satisfying the above conditions. Let $h_{1} \in \mathbb{Q}[t]$ be such that the equation $h_{1}(t)=t_{i}$ for $i=1, \ldots, n$ has no solutions. Clearly it is enough to show the existence of our polynomial for the first row in the system (5.5). Therefore, consider the system $A\left(t_{1}\right) Y_{1}^{4}=A\left(h_{1}\left(T_{1}\right)\right), B\left(t_{1}\right) Y_{1}^{6}=B\left(h_{1}\left(T_{1}\right)\right)$. If $A\left(t_{1}\right) B\left(t_{1}\right)=0$, then this system has finitely many rational solutions, and we can find $h_{2} \in \mathbb{Q}[t]$ such that $h=h_{1} \circ h_{2}$ is as desired. Now, assume that $A\left(t_{1}\right) B\left(t_{1}\right) \neq 0$. If $\left(A\left(h_{1}\left(T_{1}\right)\right) / A\left(t_{1}\right)\right)^{3} \neq\left(B\left(h_{1}\left(T_{1}\right)\right) / B\left(t_{1}\right)\right)^{2}$, then our system has at most $3 \operatorname{deg}\left(A \circ h_{1}\right)+2 \operatorname{deg}\left(B \circ h_{1}\right)$ solutions in $\mathbb{Q}$ and so there exists $h_{2} \in \mathbb{Q}[t]$ such that $h=h_{1} \circ h_{2}$ does the job. If $\left(A\left(h_{1}\left(T_{1}\right)\right) / A\left(t_{1}\right)\right)^{3}=\left(B\left(h_{1}\left(T_{1}\right)\right) / B\left(t_{1}\right)\right)^{2}$, the problem reduces to the examination of the curve $C: Y_{1}^{2}=H\left(T_{1}\right)$, where $H$ is a polynomial such that $A\left(h_{1}\left(T_{1}\right)\right) / A\left(t_{1}\right)=H\left(T_{1}\right)^{2}, B\left(h_{1}\left(T_{1}\right)\right) / B\left(t_{1}\right)=$ $H\left(T_{1}\right)^{3}$. If $H$ were the square of another polynomial, the family $\left\{\mathcal{E}_{t}\right\}_{t \in \mathbb{Q}}$ would be related to a split elliptic surface $\mathcal{E}$, which contradicts the assumption. Therefore, $H$ is not a square. Now, there exists a polynomial $h_{2}$ such that the genus of the curve $C^{\prime}: Y_{1}^{2}=H\left(h_{2}\left(T_{1}\right)\right)$ is $\geq 2$. From the Faltings theorem there are only finitely many rational points on $C^{\prime}$; so after a polynomial change of variable, we obtain a polynomial satisfying all the required conditions. Applying this reasoning to the second, $\ldots, n$th equation in (5.5) we obtain the statement of the theorem.

In view of the above theorem, a natural question arises:

Question 5.7. Is condition (2) of Conjecture 5.4 equivalent to Conjecture 5.5?

Acknowledgments. I would like to thank the anonymous referee for his valuable comments and Professors A. Schinzel and K. Rusek for their remarks improving the presentation.

\section{References}

[1] A. Bremner, Some simple elliptic surfaces of genus zero, Manuscripta Math. 73 (1991), 5-37.

[2] I. Connell, APECS: Arithmetic of Plane Elliptic Curves, ftp.math.mcgill.ca/pub/ apecs/.

[3] G. Faltings, Endlichkeitssätze für abelsche Varietäten über Zahlkörpern, Invent. Math. 73 (1983), 349-366.

[4] M. Kuwata and L. Wang, Topology of rational points on isotrivial elliptic surfaces, Int. Math. Res. Not. 1993, no. 3, 113-123. 
[5] E. Manduchi, Root numbers of fibers of elliptic surfaces, Compos. Math. 99 (1995), $33-58$.

[6] B. Mazur, The topology of rational points, Experiment. Math. 1 (1992), 35-45.

[7] L. J. Mordell, Diophantine Equations, Academic Press, London, 1969.

[8] R. Munshi, Density of positive rank fibers in elliptic fibrations, J. Number Theory 125 (2007), 254-266.

[9] K. Oguiso and T. Shioda, The Mordell-Weil lattice of a rational elliptic surface, Comment. Math. Univ. St. Pauli 40 (1991), 83-99.

[10] D. E. Rohrlich, Variation of the root number in families of elliptic curves, Compos. Math. 87 (1993), 119-151.

[11] T. Shioda, On the Mordell-Weil lattices, Comment. Math. Univ. St. Pauli 39 (1990), 211-240.

[12] J. Silverman, The Arithmetic of Elliptic Curves, Springer, New York, 1986.

[13] R. F. Whitehead, A rational parametric solution of the indeterminate cubic equation $z^{2}=f(x, y)$, J. London Math. Soc. 40 (1944), 68-71.

Institute of Mathematics

Jagiellonian University

Reymonta 4

30-059 Kraków, Poland

E-mail: Maciej.Ulas@im.uj.edu.pl

Received on 14.11.2006

and in revised form on 20.6.2007 